\title{
Absolute mass of photons based upon the Hamiltonian of energy content
}

Sunny Kumar ( $\nabla$ sunnykumar40@gmail.com )

Aryabhatasya Academy of Mathematics https://orcid.org/0000-0001-6995-4361

\section{Short Report}

Keywords: inertial mass-energy, vis-viva, vis-mortua, quantum mass-energy factor

Posted Date: July 8th, 2021

DOI: https://doi.org/10.21203/rs.3.rs-426760/v3

License: (9) This work is licensed under a Creative Commons Attribution 4.0 International License. Read Full License 
Title: Absolute mass of photons based upon the Hamiltonian of energy content

Author: Sunny Kumar

Affiliations: Aryabhatasya Academy of Mathematics (A.A.M)

Address: Near Jagdamba Gas Agency/Opposite Devrishi apartment, Village-Danada, IT Park, Sahastradhara Road, Dehradun, PIN Code-248013, Uttarakhand (India)

E-mail: sunnykumar40@gmail.com

Abstract: In this article, the mass-energy equivalence is analyzed based on the total energy content \& inertia of a body. The analysis included quantum energy formalization with relativity. The finding suggests that photons have absolute mass to justify their momentum.

Keywords: inertial mass-energy, vis-viva, vis-mortua, quantum mass-energy factor

\section{Declarations:}

Funding: Not applicable

Conflict of interest: Author has no conflict of interest.

Availability of data and material: Not applicable

Code availability: Not applicable 


\section{Introduction}

Albert Einstein raised the question that does the inertia of a body depends upon its energy content?[1] and he concluded that if a body gives off the energy $E$ in the form of radiation, its mass diminished by $E / c^{2}$ (where $c$ is the speed of light). But here, we ask the question again in its reversal form that does the energy content of a body depend upon its inertia? It follows because of my recent work on de Broglie's hypothesis on the matter-waves,[2] in which I derived an equation of total energy (Hamiltonian) of a particle having rest (absolute) mass $m$ and relative velocity $v$ and a frequency $f$ because of matter-wave observed by an observer (in a relative frame of reference).

If $\beta=\frac{v}{c} \& \alpha=\frac{1}{\gamma}=\sqrt{1-\beta^{2}}$ then $\alpha m c^{2}+\left(\frac{1}{2} m v^{2}+h f\right)=m c^{2}$

$\sum E=m c^{2}$

Now we know that Einstein never considers a quantum-energy in his theory of relativity. So how can it appear in his theory of special relativity? To analyze this, let have a look at his above paper in which he gives the change in kinetic energy by $\Delta T$,

$\Delta T=(\gamma-1) m c^{2}$

If we expand the above in binomial terms, we get

$\Delta T=m c^{2}\left(\frac{1}{2} \beta^{2}+\frac{3}{8} \beta^{4}+\frac{5}{16} \beta^{6}+\frac{35}{126} \beta^{8}+\cdots \ldots \ldots \ldots \ldots\right)$

Now if we see his paper, he smartly hides the quantum energy by neglecting the fourth and higher orders and approximate the result for low velocity as $\Delta T \approx \frac{\beta^{2}}{2} m c^{2}=\frac{1}{2} m v^{2}$. Let the sum of all binomial terms (excluding first) is $Q$, then $\Delta T=\frac{\beta^{2}}{2} m c^{2}+\mathrm{Q}$. If $v \ll c$, then Einstein approximation holds good. But for velocities near $c$ the quantity $Q$ grows fast and creates catastrophe at $c$. The term Q demands explanations because the theory 
of special relativity is the study of high velocities. And that is the point where I build my argument in my recent work on the de Broglie hypothesis. If we denote inertial energy by $E_{\alpha}$, kinetic energy by $E_{k}$ and quantum-energy of matter-wave by $E_{q}$, then work-done (by the force, which is the reason behind the motion) in the center of mass frame is given by the change in inertial energy, $(1-\alpha) m c^{2}=\left(E_{k}+E_{q}\right)$, and the work done (by the same force as stated above) in the relative frame is given by the change in kinetic energy by equation (1.2), which can be expressed as, $(\gamma-1) m c^{2}=\gamma\left(E_{k}+E_{q}\right)$, we can see that both are an equivalent statement.

\section{Vis-Viva \& Vis-Mortua [3]}

The competition between two physical quantities (momentum \& kinetic energy) to describe the changes in nature rise in late 1650 by two sides of philosophers. One side leaded by German mathematician Gottfried Leibniz with Emilie du Chatelet for vis-viva (energy). And the defendants of momentum led by Rene Descartes of France \& Newton himself. Descartes belief that God, the general cause of all motion in the universe, preserves the same quantity of motion and rest put into the world at the time of creation. The measurement of this quantity is termed as momentum, $P=m v$. While Leibniz objected and argued that the quantity which remains absolute and indestructible is not momentum but vis-viva. Now, these two principles are understood as complementary together. But we can see that the 'living force' (vis-viva) gets more attention from philosophers over the 'dead force' (vis-mortua). The dead force or as I like to call it sleeping force/energy is the concept, which not got eyes. But I find it useful in my analysis. It can be interpreted as inertial mass/energy. On the other hand, the living force gets limited to kinetic energy. If we see the equation (1.1) then, we can compare the term

$\left(\alpha m c^{2}\right)$ as the sleeping energy $\&$ the sum of the remaining two terms $\left(\frac{1}{2} m v^{2}+h f\right)$ as the living/awaken energy. And whenever there is an increment in relative motion then the 
sleeping thing gets awaken and becomes a living thing and vice-versa. Now we can ask that what is Newton's idea of force? "It is an agent, which produces a change in nature". The reason by which energy flows in an isolated system. The force can only change the inertia of a body and can't change the absolute or overall energy when applied to that body.

\section{Factors for energy in special relativity}

The equivalence factor for inertial energy is $\alpha$ and for kinetic energy is $\frac{\beta^{2}}{2}$. We can derive the factor of equivalence for a quantum energy $E_{q}$ by equation (1.1), $E_{q}=\left(1-\alpha-\frac{\beta^{2}}{2}\right) m c^{2}$

$E_{q}=A m c^{2}$

Where $A$ is the equivalence factor for quantum energy, which has the value of $\left(1-\alpha-\frac{\beta^{2}}{2}\right)$.

The table for values of these factors is given below as relative velocity increases 0 to $\mathrm{c}$. In this table, we can see that how the quantum energy of matter-wave in a moving body evolves with kinetic energy and create a catastrophe in the relative energy for photons.

\begin{tabular}{|l|l|c|c|c|c|}
\hline \multicolumn{1}{|c|}{$v$} & $\beta$ & $\gamma$ & $\alpha$ & $\frac{\beta^{2}}{2}$ & $A$ \\
\hline 0 & 0 & 1 & 1 & 0 & 0 \\
\hline 29979245.8 & 0.1 & 1.0050378152 & 0.9949874371 & 0.0050000000 & 0.0000125629 \\
\hline 59958491.6 & 0.2 & 1.0206207261 & 0.9797958971 & 0.0200000000 & 0.0002041029 \\
\hline 89937737.4 & 0.3 & 1.0482848367 & 0.9539392014 & 0.0450000000 & 0.0010607986 \\
\hline 119916983.2 & 0.4 & 1.0910894511 & 0.9165151389 & 0.0800000000 & 0.0034848611 \\
\hline 149896229 & 0.5 & 1.1547005383 & 0.8660254037 & 0.1250000000 & 0.0089745963 \\
\hline 179875474.8 & 0.6 & 1.2500000000 & 0.8000000000 & 0.1800000000 & 0.0200000000 \\
\hline 209854720.6 & 0.7 & 1.4002800840 & 0.7141428428 & 0.2450000000 & 0.0408571572 \\
\hline 239833966.4 & 0.8 & 1.6666666667 & 0.6000000000 & 0.3200000000 & 0.0800000000 \\
\hline 269813212.2 & 0.9 & 2.2941573387 & 0.4358898943 & 0.4050000000 & 0.1591101057 \\
\hline$c$ & 1 & $\infty$ & 0 & 0.5000000000 & 0.5000000000 \\
\hline
\end{tabular}

Table 1. The values of relative factors, when velocity is increasing as $v: 0 \rightarrow c$ 


\section{Photons}

The relative energy is given by the expression, $E_{\gamma}=\gamma m c^{2}$, if we manipulate this equation, then $\left(E_{\gamma}\right)^{2}=\left(m c^{2}\right)^{2}+\left(P_{\gamma} c\right)^{2}$, where $P_{\gamma}=\gamma m v$

$\left(\gamma m c^{2}\right)^{2}=\left(m c^{2}\right)^{2}+(\gamma m v c)^{2}$

$E^{2}=\left(m c^{2}\right)^{2}=\left(\alpha m c^{2}\right)^{2}+(m v c)^{2}$

And it is not mathematically right to derive, the total energy $E$ of photons by equation (4.1), because it is relative energy not the total energy of the photon. The total energy of a photon can be measure by equation (4.2) only. Obtaining momentum from equation (4.1) by ignoring $\gamma$ term is a serious mathematical mistake, which is defiantly an obstacle for quantum energy to get formalize with relativity. It's just equivalent to saying that infinity or zero is equal to some real number. A photon has all its inertial (sleeping) energy in the awaken (vis-viva) state and contains an absolute mass, which causes momentum. The Lorentz transformation declares that the relative mass energy of photons must be infinite to hold the second postulate of special relativity. The rest mass notation $m_{0}$ for photons create confusion and destroy the beauty of relativity.[4]

\section{Conclusion:}

From equation (1.1) it directly follows that if a body absorbs any radiation of frequency $f$ (in Hertz), then its absolute mass get increases by $2 h f / c^{2}$ or $\left(1.4745 \times 10^{-50} f\right) k g$. The fact that mass energy withdraws from a body becomes the energy of radiation makes no difference, so we can conclude that the inertia of a body is the measure of its energy content and vice-versa. 


\section{Application}

The above theory can make huge application in both theoretical \& engineering fields. Though for the moment I only lay down two of its direct engineering applications.

(i) To develop the technology, which can be used in the defenses of atomic warfare

(ii) In the field of medical diagnosis \& treatments

If I elaborate the second one then as we can see that we are using the radiation in medical devices like X-rays, CT-scan \& radiation therapies in the treatments of disease like cancer. In these practices we consider only the wave aspect of the radiation. But as we saw in above analysis that radiations have absolute mass associated with the aspect of waves. This new information can revolutionize the field of such practices.

\section{References:}

[1] Einstein A., Does the Inertia of a Body depend upon its energy content? (Annalen der Physik), 18:639, (1905) Link to article

[2] Kumar S., A mathematical analysis of de Broglie hypothesis and general solution for quantization to linear momentum and mass, (A.A.M., Dehradun), pp. 13-17, (2020) Link to $\underline{\text { article }}$

[3] Iltis C., Leibniz and the vis-viva controversy, (The History of Science Society, Chicago), vol. 62, No.1 pp. 21-35, (Spring 1971), Link to article

[4] Okun L. B., The concept of mass (mass, energy, relativity), (Institute of Theoretical and Experimental Physics, Moscow), Usp. Fiz. Nauk 158, pp. 511-530 (1989) Link to article 